\title{
Minimal detectable difference of the finger and wrist range of motion: comparison of goniometry and 3D motion analysis
}

\author{
Lisa Reissner ${ }^{1 *}$ (D), Gabriella Fischer ${ }^{2}$, Renate List ${ }^{2,3}$, William R. Taylor ${ }^{2}$, Pietro Giovanoli ${ }^{1}$ and Maurizio Calcagni ${ }^{1}$
}

\begin{abstract}
Background: The measurement of finger and wrist range of motion (ROM) is of great importance to clinicians when assessing functional outcomes of therapeutic interventions and surgical procedures. The purpose of the study was to assess the repeatability of ROM measurements of the hand joints with manual goniometer and 3D motion capture system and to calculate the minimal detectable difference for both methods.

Methods: Active finger and wrist joints ROM of 20 healthy volunteers were assessed using a manual goniometer and 3D motion capture system. Minimal detectable difference (MDD) and standard error of measurement (SEM) were calculated for both measurement systems and compared within the same task. Maximal ROM of all joints was registered twice on two different days to evaluate the test-retest repeatability. The intraclass correlation coefficients (ICC) was calculated and examined to determine if reliability $\geq 0.70$ existed.
\end{abstract}

Results: MDD for the 3D motion capture was between 5 and $12^{\circ}$ except for the metacarpophalangeal joint (MCP) 1, interphalangeal joint (IP), and MCP5. SEM values lay between 2 and $4^{\circ}$ for all joints except for the MCP5, IP, and MCP1. For the goniometric measurements, MDD and SEM were between $12-30^{\circ}$ and $4-11^{\circ}$, respectively. The reliability criterion (ICC > 0.7) was achieved for the ROM measurement with the 3D motion capture system for $94 \%$ of the joints and in only $65 \%$ of the joints with the manual goniometer.

Conclusions: Joint ROM assessed with 3D motion analysis showed higher test-retest agreement demonstrating overall better repeatability for this method. Because of the smaller measurement error, the 3D motion capture system has a smaller MDD. Only individual test-rest differences bigger than the MDD can be considered as real changes, and therefore, in an experimental situation, the use of a more precise measurement method can greatly reduce the number of subjects needed for a statistical significance. Goniometer measurements of some joints should be carefully interpreted, due to a low repeatability and reliability.

Trial registration: This study is approved by the Ethical Committee Zurich (Kek-ZH-Nr: 2015-0395).

Keywords: Manual goniometer, 3D motion capture, Range of motion, Motion analysis, Minimal detectable difference

\footnotetext{
* Correspondence: lisa.reissner@usz.ch

${ }^{1}$ Division of Plastic Surgery and Hand Surgery, University Hospital Zurich,

University of Zurich, Raemistrasse 100, 8091 Zurich, Switzerland

Full list of author information is available at the end of the article
}

C The Author(s). 2019 Open Access This article is distributed under the terms of the Creative Commons Attribution 4.0 International License (http://creativecommons.org/licenses/by/4.0/), which permits unrestricted use, distribution, and reproduction in any medium, provided you give appropriate credit to the original author(s) and the source, provide a link to the Creative Commons license, and indicate if changes were made. The Creative Commons Public Domain Dedication waiver (http://creativecommons.org/publicdomain/zero/1.0/) applies to the data made available in this article, unless otherwise stated. 


\section{Introduction}

The measurement of finger and wrist postures is one of the important parameters for the clinicians when assessing the outcomes of therapeutic interventions and compare them. Joint angular measurements are also essential for hand therapists to record the progress of rehabilitation. It is therefore important for clinicians and researchers to have complete and relevant information on the accuracy, repeatability, and reliability of these measurements. While the manual goniometer is commonly used in clinical practice as a tool to measure joint angles, 3D motion capture systems are increasingly applied in research to measure hand motion [1-5]. Moreover, 3D motion capture systems allow the dynamic evaluation of all hand joints simultaneously [6-8]. They determine the position of skin markers highly accurate. The main advantages of the manual goniometry are that it is cheap, fast, and does not require data post-processing or joint angle calculations, and the main drawback is that it relies on rater's performance for the quality of measurements. For evaluative instruments that are used to measure changes in the same subject over time, the ability to detect minimal clinically important differences is essential. Hence, it is fundamental to know the size of the measurement error is required not only for the selection of the appropriate measurement tool, but also for the interpretation of the data and the comparison between different studies.

Trained therapists generally have adequate intrarater reliability for the measurement of wrist and finger postures; however, some joints are easier to assess [9-12]. When standard goniometry is used, variability between 2 and $7^{\circ}$ occurs in joint angle measurements of the hand [13-15]. The validation of goniometer measurement was done in splinted positions, which means that the force applied on the joint by the examiner was neutralized. This is not the case in real life where joints are examined looking for the actual angles and not a predefined one $[16,17]$. Moreover, there are few studies comparing manual goniometer measurement and 3D motion capture, but none of them taking into account all the joints of the hand [16].

Sample size calculation (power) is a standard requirement for high-quality studies. Minimal detectable difference (MDD) and standard error of measurement (SEM) are among the most important parameters for its calculation. If we can reduce them, this will result in a smaller sample size.

Therefore, the aim of this study was to assess the repeatability of ROM of the hand joints with manual goniometer and 3D motion capture system and to calculate the minimal detectable difference and the standard error of measurement for both methods.

\section{Material and methods}

\section{Participants and protocol}

Finger and wrist joints motion of 20 healthy right-hand dominant volunteers (ten men, ten women) with mean age 28 years (SD 4.7) were assessed with 3D motion capture system and measured with manual goniometer. In order to assess the test-retest repeatability for both methods, each participant was tested on two different days. The same hand surgeon performed all goniometric measurements, and the same examiner placed the skin markers in both sessions. The local ethics committee approved the study, and all participants provided written informed consent for their data to be used for this analysis (Kek-ZH-Nr: 2015-0395).

\section{Manual goniometric measurements}

The protocol of the goniometric measurement followed the recommendations of the American Society for Hand Therapists for the wrist joint and the finger joints [17-19]. Each volunteer placed their elbow on the table with the forearm in neutral position. Dorsal placement of a plastic goniometer (Zimmer ${ }^{\odot}$ ) on the wrist was applied. For the measurement of the distal interphalangeal joint (DIP), raters instructed patients to maximal extend the metacarpophalangeal joint (MCP) while maximal flexion of the proximal interphalangeal (PIP) joint and DIP joint, making a hook fist. During pronation and supination of the forearm, the volunteer was sitting with the shoulder in $0^{\circ}$ of flexion, extension, abduction, and rotation so that the upper arm was close to the side of the body. The elbow was in $90^{\circ}$ of flexion, and the goniometer was placed just proximal to the radial and ulnar styloid process while performing maximal pronation and supination.

\section{D motion capture system and setup}

An optoelectronic motion capture system consisting of 11 fixated infrared cameras $\left(\mathrm{VICON}^{-} \mathrm{MX}^{-}+\right.$and $\mathrm{VICON}^{\odot} \mathrm{MX} 3$ motion capture system, Oxford Metrics Ltd., UK) and the corresponding software VICON ${ }^{\oplus}$ Nexus (version 2.3) were used for data collection. The capture volume was approximately $50 \times 50 \times 50 \mathrm{~cm}^{3}$, and the cameras were positioned such that the markers were always visible by at least two cameras, avoiding hiding of markers (Fig. 1). The cameras have a resolution of $659 \times 493$ pixel, and recordings were carried out with a frequency of $100 \mathrm{~Hz}$.

In total, 46 reflective markers were placed at specific positions on the finger, wrist, and forearm (Fig. 2). The three markers on the elbow are located on the lateral and medial epicondyle as well as proximal of the olecranon. The spherical markers at the elbow and forearm 


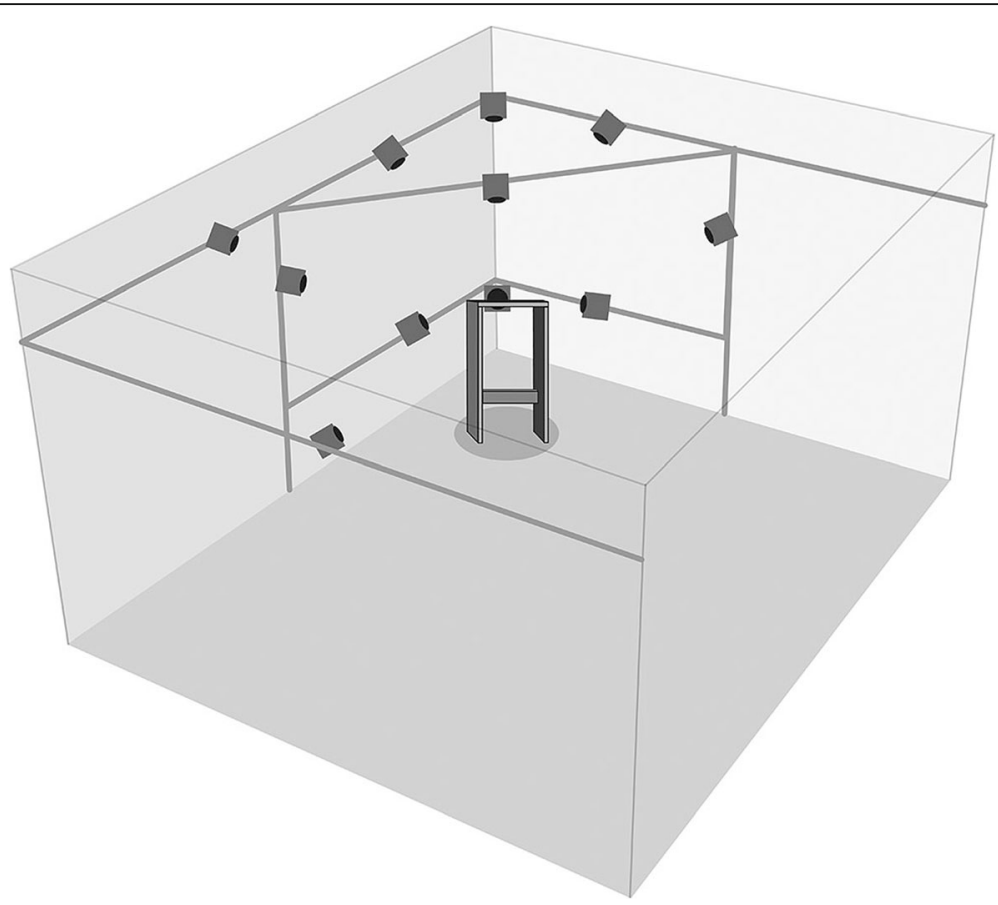

Fig. 1 Camera setup

had a diameter of $9 \mathrm{~mm}$ and $5 \mathrm{~mm}$, respectively. For the hand and fingers, hemispherical markers with a diameter of $3 \mathrm{~mm}$ were chosen. We attached the markers with a skin-compatible adhesive tape.

\section{Motion tasks}

First, a natural reference position with the hand lying on a flat surface and a $40^{\circ}$ wedge between the second finger and the thumb was recorded. Afterwards, each volunteer performed a set of basic motion tasks:

- Pronation-supination $(\mathrm{P} / \mathrm{S})$ of the forearm

- Flexion-extension (F/E) and radial-ulnar deviation $(\mathrm{R} / \mathrm{U})$ and of the wrist

- Combined F/E in the MCP, PIP, and DIP joints of the long fingers (make a fist)

- F/E of the PIP and DIP joints of the long fingers (without movement in the MCP joints)

- F/E of the thumb

The tasks aimed to detect joint ROM in a single movement plane. Furthermore, they were used to calculate functional joint axes and centers. Each trial started and ended with the hand in a neutral position and consisted of three cycles (e.g., flexion-extension-flexion-extension-flexion-extension). Five valid repetitions of each task were recorded per session.

\section{Data processing and data analysis}

For the kinematic description of the hand, 18 segments were defined and considered as rigid bodies. At least three markers per segment are needed to allow kinematic analysis in all three movement planes. The kinematic model of the fingers was based on the assumption that only motion around the flexion axis is possible for the PIP and DIP joints. Therefore, kinematics of the long fingers could be assessed with a reduced marker concept using only two longitudinally aligned markers per segment. The F/E angles were calculated by means of the vectors between the markers of each finger segment similar to Metcalf et al. but with a marker proximal or distal of the joint defining the segment vector instead of markers on the joint [20,21].

The kinematic evaluation of the distal radioulnar joint, the wrist joint, and the thumb joints was based on marker clusters. As described in List et al., the rotation of a segment relative to a static reference position was estimated during the dynamic trials using a least squares fit [22]. Then, joint kinematics was expressed as the relative rotation matrix of the distal segment relative to the proximal segment. To determine the joint centers and joint axes from specific calibration movements, a functional approach from List et al. was adapted to the thumb and wrist [22]. Joint rotations were calculated according to Grood and Suntay and in accordance with the 


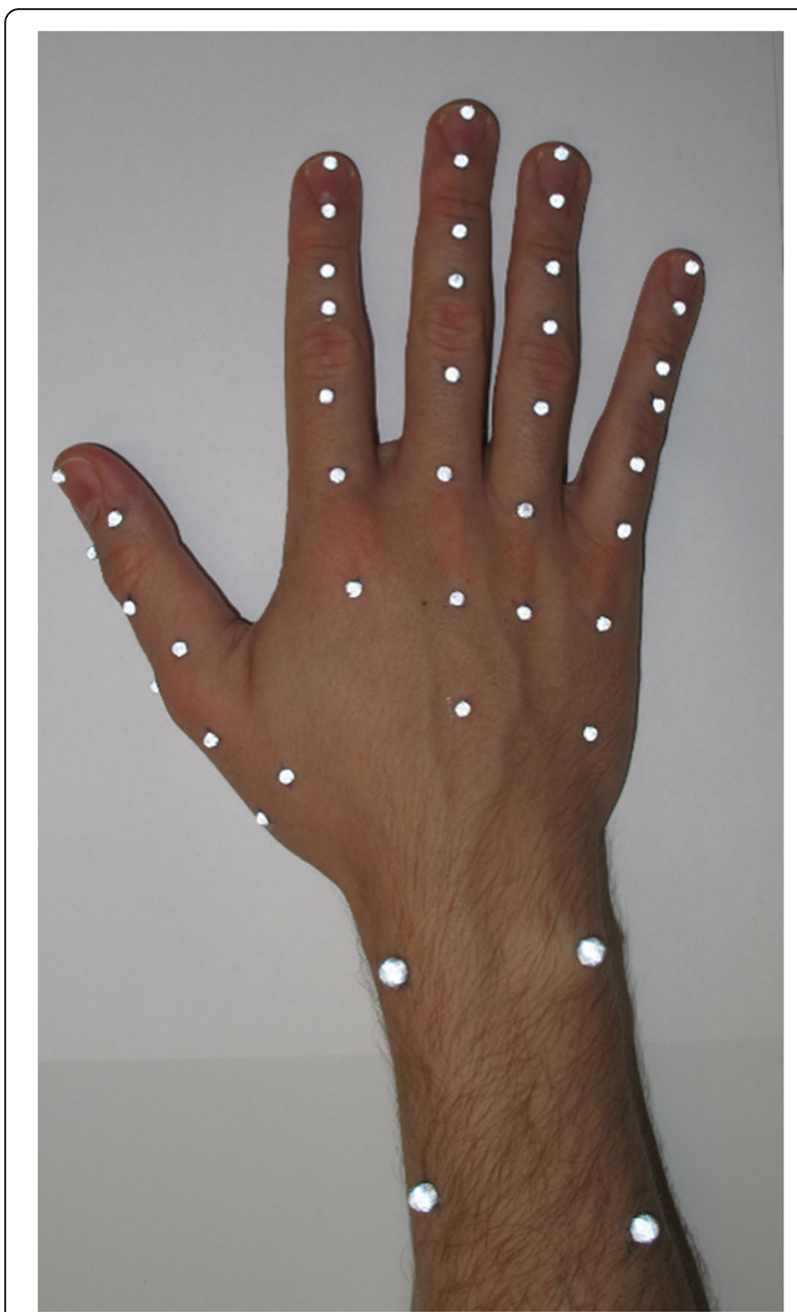

Fig. 2 Marker position of the fingers and the wrist
It is recommended that ICC values need to be greater than or equal to 0.70 to be considered acceptable as a clinically meaningful measurement tool [25]. According to de Vet et al., the SEM represents measurement error and equals the square root of the variance of differences [26]. Only changes of the ROM that exceed the variability induced by the method can be regarded as real changes [27]. Therefore, based on the SEM, the minimal detectable difference (MDD) of both methods was calculated. In addition, the percentage of subjects with absolute test-retest differences below $5^{\circ}$ and $10^{\circ} \quad\left(\mathrm{PD}<5^{\circ} / \mathrm{PD}<10^{\circ}\right)$ were determined for both methods, respectively.

\section{Statistical analysis}

Two-tailed $t$ tests $(p \leq 0.05)$ were performed to compare motion analysis and goniometry regarding the mean test-retest difference $\left(\right.$ meanD $_{\mathrm{V}}$ vs. mean $\left.\mathrm{D}_{\mathrm{G}}\right)$ as well as the average ROM $\left(\mathrm{Mean}_{\text {romv }}\right.$ vs. Mean romG $\left._{\text {ro }}\right)$. For a healthy population, we assumed ROM to be constant over time. Therefore, $\operatorname{DIFF}_{G}$ and $\mathrm{DIFF}_{\mathrm{V}}$ are considered as an estimation of measurement error, and a limit of $5^{\circ}$ was set based upon the accuracy of the manual goniometer shown to be around $5^{\circ}$ in literature [28]. The null hypotheses were that:

$\left(\mathrm{H}_{01}\right)$ Within the same method, meanD equals zero, $\left(\mathrm{H}_{02}\right)$ DIFF lies within $5^{\circ}$, and $\left(\mathrm{H}_{03}\right)$ the $\mathrm{ROM}$ is equivalent when measured with the goniometer or the motion capture system.

$\mathrm{H}_{01}$ was rejected, if the absolute value of the ratio of meanD and standard deviation of difference (SDD) exceeds the $5 \%$ level of agreement $(|\mathrm{T}|>1.96 ; p \leq 0.05)$. $\mathrm{H}_{02}$ was rejected, if the estimated precision represented by the SEM was $>5^{\circ}$.

standards defined by the International Society of Biomechanics [23, 24].

After checking recorded data for errors, the data were summarized using descriptive statistics. All analyzed parameters and their abbreviations are defined in Table 1. For all abbreviations of these parameters, a subscripted character $G$ refers to the goniometer measurement and subscripted character $\mathrm{V}$ refers to the analysis by means of the motion capture system.

For the dynamic trials recorded by the motion capture system, the minimum and maximum joint angle (e.g., maximum flexion and extension position) was determined for each trial and averaged within the five trials of the same session to obtain the ROM. The individual test-retest difference (DIFF) of the ROM was determined for each subject. To quantify the test-retest repeatability of the ROM, SEM and intra-class correlation coefficient (ICC) were calculated within the same task and method for all joints.

\section{Results}

For the 3D motion capture system, no valid joint angle could be calculated for four subjects at the radioulnar joint and for one subject at the MCP5 joint due to issues with visibility of markers or a lost marker at the elbow. Furthermore, one subject had a misplaced marker on the thumb, affecting MCP1 calculations, and one subject had a shifted marker affecting MCP4 and MCP5 angle calculations. Therefore, these values had to be excluded from further data analysis after visual inspection of the recorded data. The available data are reported in the second column of Table 2.

There was a wide range of maximum ROM among the healthy subjects $\left(\mathrm{SD}_{\text {romv }} 9^{\circ}, \mathrm{SD}_{\text {romG }} 10^{\circ}\right)$ (Tables 3 and 4). The two-tailed $t$ test revealed significant $(p<$ 0.05 ) differences of the $M^{2} n_{\text {rom }}$ derived from the different methods only for the pronation-supination movement of the radioulnar joint. For this joint, 
Table 1 Kinematic parameters

\begin{tabular}{|c|c|c|}
\hline Parameter & Abbreviation & Description/formula \\
\hline \multicolumn{3}{|c|}{ Measurement parameter-determined for each subject in measurement session $1+2$ (test-retest) } \\
\hline Range of motion & $\mathrm{ROM}_{\mathrm{G} 1 / \mathrm{G} 2}, \mathrm{ROM}_{\mathrm{V} 1 / \mathrm{V} 2}$ & Maximum range of motion measured within a method and session \\
\hline Test-retest difference & $\mathrm{DIFF}_{\mathrm{G}} / \mathrm{DIFF}$ & $\begin{array}{l}\text { Individual test-retest difference of } \mathrm{ROM}: \mathrm{DIFF}_{\mathrm{G}}=\mathrm{ROM}_{\mathrm{G} 1}-\mathrm{ROM}_{\mathrm{G} 2} / \\
\mathrm{DIFF}_{\mathrm{V}}=\mathrm{ROM}_{\mathrm{V} 1}-\mathrm{ROM}_{\mathrm{V} 2}\end{array}$ \\
\hline \multicolumn{3}{|c|}{ Test-retest and repeatability parameter-between test and retest within the same method over all subjects } \\
\hline Mean difference & meanD ${ }_{G} /$ meanD $D_{V}$ & Mean $\mathrm{DIFF}_{\mathrm{G}} / \mathrm{DIFF}$ \\
\hline Standard deviation of the difference & $\mathrm{SDD}_{\mathrm{G}} / \mathrm{SDD}_{\vee}$ & Standard deviation of $\mathrm{DIFF}_{\mathrm{G}} / \mathrm{DIFF}_{\mathrm{V}}$ \\
\hline Standard error of measurement & $\mathrm{SEM}_{G} / \mathrm{SEM}_{V}$ & $\begin{array}{l}\text { Estimated measurement precision according to de } \operatorname{Vet}^{24} \text { : } \\
\mathrm{SEM}=\mathrm{SDD} / \sqrt{2}\end{array}$ \\
\hline Minimal detectable difference & $\mathrm{MDD}_{\mathrm{G}} / \mathrm{MDD}_{V}$ & $\mathrm{MDD}=1.96 \times \mathrm{SDD}$ \\
\hline$\%$ within $5^{\circ}$ or $10^{\circ}$ & $\mathrm{PD}_{\mathrm{G}}<5^{\circ}$ or $<10^{\circ} / \mathrm{PD}_{V}<5^{\circ}$ or $<10^{\circ}$ & $\begin{array}{l}\text { Percentage of subjects with an absolute }\left|\mathrm{DIFF}_{\mathrm{G}}\right| /\left|\mathrm{DIFF}_{\mathrm{V}}\right| \text { smaller than } \\
5^{\circ} \text { or } 10^{\circ}\end{array}$ \\
\hline Intraclass correlation coefficient & $\mathrm{ICC}_{\mathrm{G}} / \mathrm{ICC} C_{V}$ & $\begin{array}{l}\text { Intraclass correlation coefficient }(3,1) \text { of the test-retest measurement } \\
\text { of the ROM }\end{array}$ \\
\hline \multicolumn{3}{|c|}{ Method comparison—between measurement methods } \\
\hline Mean ROM & Mean $_{\text {romG }} /$ Mean $_{\text {romv }}$ & $\begin{array}{l}\text { Mean ROM of test and retest of each subjects averaged over all } \\
\text { subjects }\end{array}$ \\
\hline Standard deviation of ROM & $\mathrm{SD}_{\text {romG }} / \mathrm{SD}_{\text {romv }}$ & Standard deviation of Mean romG $_{\text {Mean }}$ romv \\
\hline
\end{tabular}

Mean $_{\text {romG }}$ and Mean $_{\text {romV }}$ differ by almost $57^{\circ}$, with the lower values measured with the $3 \mathrm{D}$ motion capture system.

The results of the test-retest parameters are presented in Table 2. No statistically significant difference $\left(\mathrm{H}_{01}\right)$ of the $\mathrm{ROM}(p$ level $\leq 0.05)$ was found between the first and second measurement for both methods in all joints.

For the $3 \mathrm{D}$ motion capture method, $\mathrm{MDD}_{\mathrm{V}}$ lay between 5 and $12^{\circ}$ except for the MCP1, IP, and MCP5.

Table 2 Test-retest results of goniometer and 3D motion analysis measurement

\begin{tabular}{|c|c|c|c|c|c|c|c|c|c|c|c|c|c|}
\hline \multirow[b]{2}{*}{ Joint } & \multirow[b]{2}{*}{ Direction } & \multicolumn{6}{|c|}{ Motion Capture } & \multicolumn{6}{|c|}{ Goniometer } \\
\hline & & $n_{V}$ & $\begin{array}{l}\mathrm{MDD}_{V} \\
\left.{ }^{\circ}\right]\end{array}$ & $\begin{array}{l}\text { SEMV } \\
{\left[{ }^{\circ}\right]}\end{array}$ & $\mathrm{ICC}_{\mathrm{V}}$ & $\begin{array}{l}P D_{V}<5^{\circ} \\
{[\%]}\end{array}$ & $\begin{array}{l}\mathrm{PD}_{V}<10^{\circ} \\
{[\%]}\end{array}$ & $n_{G}$ & $\begin{array}{l}\mathrm{MDD}_{\mathrm{G}} \\
\left.{ }^{\circ}\right]\end{array}$ & $\begin{array}{l}\mathrm{SEM}_{\mathrm{G}} \\
\left.{ }^{\circ}\right]\end{array}$ & $I C C_{G}$ & $\begin{array}{l}\mathrm{PD}_{\mathrm{G}}<5^{\circ} \\
{[\%]}\end{array}$ & $\begin{array}{l}\mathrm{PD}_{\mathrm{G}}<10^{\circ} \\
{[\%]}\end{array}$ \\
\hline Radioulnar & $\mathrm{P} / \mathrm{S}$ & 16 & 8 & 3.0 & 0.94 & 69 & 100 & 20 & 19 & 6.8 & 0.35 & 45 & 65 \\
\hline Wrist & $\mathrm{F} / \mathrm{E}$ & 20 & 6 & 2.1 & 0.97 & 95 & 100 & 20 & 18 & 6.4 & 0.87 & 50 & 75 \\
\hline Wrist & $R / U$ & 20 & 7 & 2.6 & 0.90 & 90 & 95 & 20 & 18 & 6.5 & 0.72 & 55 & 65 \\
\hline MCP2 & $\mathrm{F} / \mathrm{E}$ & 20 & 7 & 2.7 & 0.96 & 80 & 100 & 20 & 19 & 7.0 & 0.79 & 25 & 60 \\
\hline MCP3 & $\mathrm{F} / \mathrm{E}$ & 20 & 9 & 3.1 & 0.95 & 65 & 100 & 20 & 24 & 8.8 & 0.59 & 35 & 60 \\
\hline MCP4 & $\mathrm{F} / \mathrm{E}$ & 19 & 9 & 3.4 & 0.92 & 47 & 100 & 20 & 18 & 6.6 & 0.81 & 40 & 60 \\
\hline MCP5 & $\mathrm{F} / \mathrm{E}$ & 18 & 16 & 5.7 & 0.90 & 33 & 72 & 20 & 22 & 8.0 & 0.86 & 20 & 40 \\
\hline PIP2 & $\mathrm{F} / \mathrm{E}$ & 20 & 8 & 3.0 & 0.85 & 85 & 95 & 20 & 12 & 4.2 & 0.66 & 55 & 80 \\
\hline PIP3 & $\mathrm{F} / \mathrm{E}$ & 20 & 12 & 4.2 & 0.66 & 65 & 85 & 20 & 12 & 4.3 & 0.64 & 55 & 80 \\
\hline PIP4 & $\mathrm{F} / \mathrm{E}$ & 20 & 10 & 3.8 & 0.86 & 80 & 85 & 20 & 13 & 4.6 & 0.60 & 60 & 75 \\
\hline PIP5 & $\mathrm{F} / \mathrm{E}$ & 20 & 11 & 4.0 & 0.85 & 65 & 90 & 20 & 15 & 5.5 & 0.40 & 45 & 65 \\
\hline DIP2 & $F / E$ & 20 & 9 & 3.4 & 0.92 & 65 & 90 & 20 & 14 & 5.0 & 0.79 & 45 & 80 \\
\hline DIP3 & $\mathrm{F} / \mathrm{E}$ & 20 & 9 & 3.3 & 0.92 & 60 & 100 & 20 & 18 & 6.7 & 0.75 & 30 & 60 \\
\hline DIP4 & $F / E$ & 20 & 10 & 3.5 & 0.96 & 70 & 95 & 20 & 17 & 6.3 & 0.84 & 40 & 65 \\
\hline DIP5 & $F / E$ & 19 & 11 & 4.0 & 0.94 & 58 & 95 & 20 & 14 & 4.9 & 0.86 & 60 & 85 \\
\hline IP & $\mathrm{F} / \mathrm{E}$ & 20 & 14 & 5.0 & 0.93 & 50 & 80 & 20 & 30 & 11.0 & 0.85 & 35 & 50 \\
\hline MCP1 & $\mathrm{F} / \mathrm{E}$ & 19 & 14 & 5.0 & 0.91 & 68 & 79 & 20 & 19 & 6.8 & 0.83 & 30 & 70 \\
\hline
\end{tabular}


Table 3 Range of motion (mean and SD) for goniometer and 3D motion capture measurement

\begin{tabular}{|c|c|c|c|c|c|c|c|c|c|c|c|}
\hline \multirow{4}{*}{ Joint } & \multirow{4}{*}{ Direction } & \multicolumn{4}{|c|}{ Measurement day 1} & \multirow{4}{*}{$P$ value } & \multicolumn{4}{|c|}{ Measurement day 2} & \multirow{4}{*}{$P$ value } \\
\hline & & \multicolumn{2}{|c|}{ Motion Capture } & \multicolumn{2}{|l|}{ Goniometer } & & \multicolumn{2}{|c|}{ Motion Capture } & \multicolumn{2}{|l|}{ Goniometer } & \\
\hline & & Mean $_{\text {romv1 }}$ & $\mathrm{SD}_{\text {romV1 }}$ & Mean $_{\text {romG1 }}$ & $\mathrm{SD}_{\text {romG1 }}$ & & Mean $_{\text {romv2 }}$ & $\mathrm{SD}_{\text {romv2 }}$ & Mean $_{\text {romG2 }}$ & $\mathrm{SD}_{\text {romG2 }}$ & \\
\hline & & {$\left[{ }^{\circ}\right]$} & {$\left[{ }^{\circ}\right]$} & {$\left[{ }^{\circ}\right]$} & {$\left[{ }^{\circ}\right]$} & & {$\left[{ }^{\circ}\right]$} & {$\left[{ }^{\circ}\right]$} & {$\left[{ }^{\circ}\right]$} & {$\left[{ }^{\circ}\right]$} & \\
\hline Radioulnar & $\mathrm{P} / \mathrm{S}$ & 116 & 10 & 174 & 8 & $<0.0001$ & 114 & 10 & 170 & 8 & $<0.0001$ \\
\hline Wrist & $F / E$ & 150 & 9 & 141 & 14 & 0.570 & 150 & 10 & 139 & 12 & 0.499 \\
\hline Wrist & $R / U$ & 51 & 7 & 56 & 10 & 0.663 & 51 & 6 & 58 & 10 & 0.573 \\
\hline MCP2 & $F / E$ & 109 & 10 & 114 & 12 & 0.787 & 109 & 9 & 116 & 13 & 0.663 \\
\hline MCP3 & $\mathrm{F} / \mathrm{E}$ & 114 & 10 & 114 & 11 & 0.991 & 113 & 10 & 115 & 13 & 0.902 \\
\hline MCP4 & $F / E$ & 113 & 11 & 115 & 10 & 0.883 & 110 & 10 & 119 & 14 & 0.644 \\
\hline MCP5 & $\mathrm{F} / \mathrm{E}$ & 115 & 16 & 120 & 14 & 0.795 & 112 & 13 & 123 & 19 & 0.624 \\
\hline PIP2 & $F / E$ & 118 & 6 & 109 & 6 & 0.275 & 118 & 6 & 113 & 7 & 0.617 \\
\hline PIP3 & $\mathrm{F} / \mathrm{E}$ & 119 & 6 & 109 & 6 & 0.234 & 118 & 6 & 112 & 7 & 0.487 \\
\hline PIP4 & $\mathrm{F} / \mathrm{E}$ & 122 & 7 & 109 & 6 & 0.186 & 121 & 8 & 112 & 7 & 0.411 \\
\hline PIP5 & $\mathrm{F} / \mathrm{E}$ & 105 & 7 & 101 & 5 & 0.705 & 106 & 9 & 103 & 8 & 0.775 \\
\hline DIP2 & $\mathrm{F} / \mathrm{E}$ & 80 & 10 & 84 & 9 & 0.751 & 83 & 10 & 84 & 9 & 0.899 \\
\hline DIP3 & $\mathrm{F} / \mathrm{E}$ & 93 & 9 & 95 & 12 & 0.927 & 95 & 9 & 94 & 9 & 0.921 \\
\hline DIP4 & $\mathrm{F} / \mathrm{E}$ & 84 & 13 & 87 & 12 & 0.882 & 85 & 13 & 89 & 12 & 0.833 \\
\hline DIP5 & F/E & 89 & 13 & 89 & 10 & 0.996 & 88 & 12 & 90 & 11 & 0.906 \\
\hline IP & $\mathrm{F} / \mathrm{E}$ & 93 & 14 & 103 & 23 & 0.720 & 92 & 13 & 104 & 21 & 0.643 \\
\hline MCP1 & $\mathrm{F} / \mathrm{E}$ & 72 & 15 & 71 & 14 & 0.947 & 70 & 12 & 72 & 11 & 0.964 \\
\hline
\end{tabular}

Significant difference between motion capture and goniometer measurement

Table 4 Range of motion (median and range) for goniometer and 3D motion analysis measurement

\begin{tabular}{|c|c|c|c|c|c|c|c|c|c|c|c|c|c|}
\hline \multirow{4}{*}{ Joint } & \multirow{4}{*}{ Direction } & \multicolumn{6}{|c|}{ Measurement day 1} & \multicolumn{6}{|c|}{ Measurement day 2} \\
\hline & & \multicolumn{3}{|c|}{ Motion Capture } & \multicolumn{3}{|l|}{ Goniometer } & \multicolumn{3}{|c|}{ Motion Capture } & \multicolumn{3}{|l|}{ Goniometer } \\
\hline & & Median $_{\text {romv1 }}$ & Min $_{\text {romV1 }}$ & Max ${ }_{\text {romV1 }}$ & Median $_{\text {romG1 }}$ & Min $_{\text {romG1 }}$ & Max $_{\text {romG1 }}$ & Median $_{\text {romv2 }}$ & $\mathrm{Min}_{\text {romV2 }}$ & $\operatorname{Max}_{\text {romv2 }}$ & Median $_{\text {romG2 }}$ & Min $_{\text {romG2 } 2}$ & Max \\
\hline & & {$\left[{ }^{\circ}\right]$} & {$\left[{ }^{\circ}\right]$} & {$\left[{ }^{\circ}\right]$} & {$\left[{ }^{\circ}\right]$} & {$\left[{ }^{\circ}\right]$} & {$\left[{ }^{\circ}\right]$} & {$\left[{ }^{\circ}\right]$} & {$\left[{ }^{\circ}\right]$} & {$\left[{ }^{\circ}\right]$} & {$\left[{ }^{0}\right]$} & {$\left[{ }^{\circ}\right]$} & {$\left[{ }^{\circ}\right]$} \\
\hline Radioulnar & $\mathrm{P} / \mathrm{S}$ & 117 & 101 & 136 & 172 & 160 & 190 & 114 & 99 & 137 & 170 & 156 & 182 \\
\hline Wrist & $F / E$ & 151 & 129 & 166 & 141 & 102 & 168 & 150 & 127 & 165 & 139 & 105 & 160 \\
\hline Wrist & $R / U$ & 52 & 37 & 62 & 57 & 42 & 82 & 52 & 40 & 62 & 58 & 44 & 84 \\
\hline MCP2 & $F / E$ & 108 & 91 & 132 & 112 & 90 & 138 & 108 & 94 & 127 & 116 & 96 & 136 \\
\hline MCP3 & $F / E$ & 112 & 98 & 137 & 115 & 98 & 134 & 112 & 99 & 133 & 115 & 96 & 138 \\
\hline MCP4 & $F / E$ & 112 & 100 & 138 & 115 & 96 & 134 & 107 & 93 & 130 & 119 & 100 & 148 \\
\hline MCP5 & $F / E$ & 116 & 90 & 151 & 121 & 98 & 151 & 110 & 93 & 137 & 123 & 94 & 162 \\
\hline PIP2 & $F / E$ & 118 & 110 & 132 & 108 & 98 & 118 & 119 & 109 & 128 & 113 & 102 & 132 \\
\hline PIP3 & $\mathrm{F} / \mathrm{E}$ & 120 & 109 & 129 & 108 & 98 & 118 & 118 & 108 & 131 & 112 & 102 & 126 \\
\hline PIP4 & $\mathrm{F} / \mathrm{E}$ & 123 & 110 & 135 & 108 & 100 & 120 & 122 & 105 & 133 & 112 & 100 & 122 \\
\hline PIP5 & $\mathrm{F} / \mathrm{E}$ & 105 & 90 & 120 & 102 & 84 & 110 & 106 & 83 & 119 & 103 & 90 & 114 \\
\hline DIP2 & $\mathrm{F} / \mathrm{E}$ & 79 & 65 & 98 & 84 & 66 & 104 & 84 & 66 & 100 & 84 & 62 & 96 \\
\hline DIP3 & $\mathrm{F} / \mathrm{E}$ & 92 & 77 & 107 & 94 & 70 & 114 & 98 & 81 & 109 & 94 & 76 & 108 \\
\hline DIP4 & $F / E$ & 87 & 58 & 103 & 89 & 68 & 114 & 85 & 61 & 105 & 89 & 64 & 104 \\
\hline DIP5 & $F / E$ & 93 & 61 & 107 & 91 & 74 & 108 & 88 & 70 & 108 & 90 & 70 & 110 \\
\hline $\mathbb{P}$ & $\mathrm{F} / \mathrm{E}$ & 93 & 67 & 114 & 101 & 66 & 152 & 92 & 58 & 111 & 104 & 70 & 160 \\
\hline MCP1 & $\mathrm{F} / \mathrm{E}$ & 72 & 47 & 110 & 70 & 50 & 104 & 70 & 50 & 97 & 72 & 56 & 98 \\
\hline
\end{tabular}




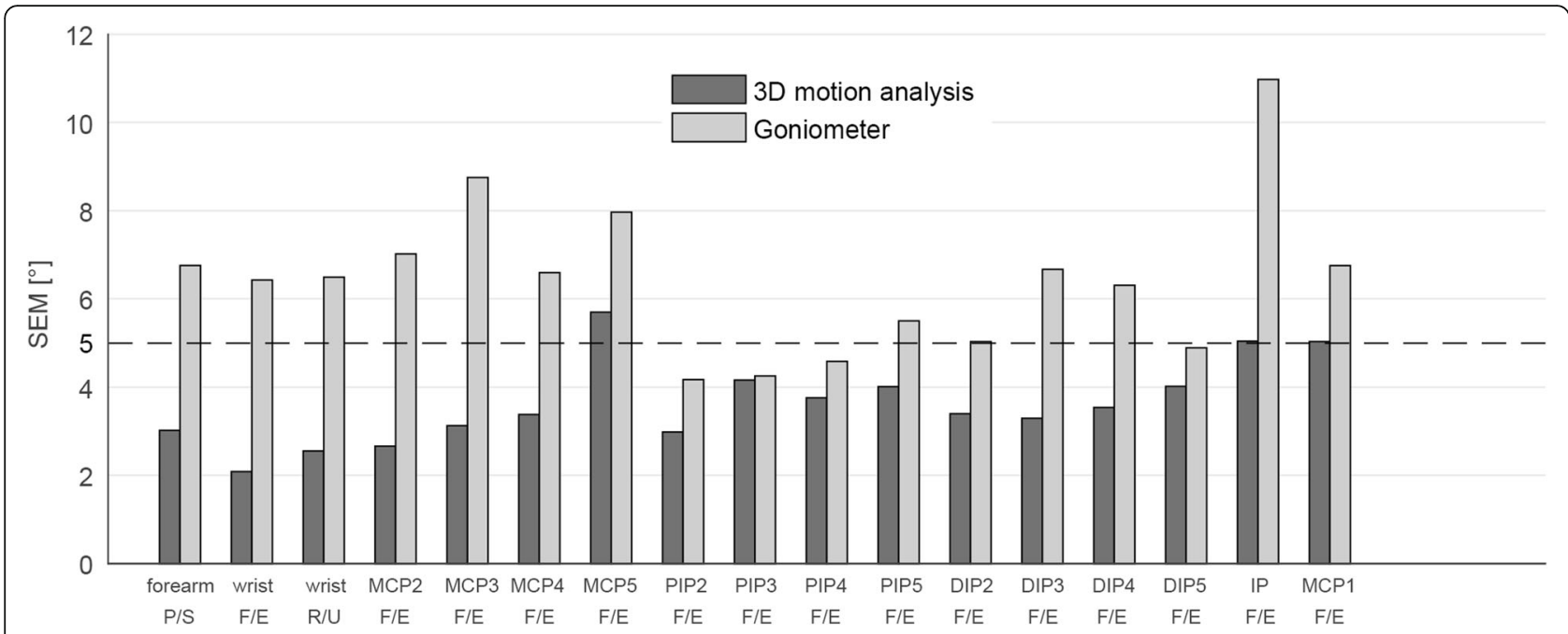

Fig. 3 SEM of $3 D$ motion analysis and goniometer of all joints

For the goniometric measurements, $\mathrm{MDD}_{\mathrm{G}}$ was between 12 and $30^{\circ}$.

The observed precision of the measurement represented by the SEM is displayed in Fig. 3 and Table 2. $\mathrm{SEM}_{\mathrm{V}}$ lied below the limit of $5^{\circ}$ for all joints except for the MCP5 $\left(\mathrm{SEM}_{\mathrm{V}} 5.7^{\circ}\right)$, IP $\left(\mathrm{SEM}_{\mathrm{V}} 5.0^{\circ}\right)$, and $\mathrm{MCP1}\left(\mathrm{SEM}_{\mathrm{V}} 5.0^{\circ}\right)$. For the goniometric measurements, $\mathrm{SEM}_{\mathrm{G}}$ exceeds the limit of $5^{\circ}\left(\mathrm{SEM}_{\mathrm{G}} 5.0-\right.$ $\left.11.0^{\circ}\right)$ in all joints, except for PIP2-4 and DIP5 $\left(\mathrm{SEM}_{\mathrm{G}}\right.$ $\left.4.2-4.9^{\circ}\right)$.

ICC ranged from 0.35 to $0.87\left(\mathrm{ICC}_{\mathrm{G}}\right)$ and 0.66 to 0.97 $\left(\mathrm{ICC}_{\mathrm{V}}\right)$ for the goniometric and 3D motion capture measurements, respectively (Table 2). Six out of 17 joints (radioulnar, MCP3, PIP2-5) did not achieve the reliability criterion with the manual goniometer. In comparison, the $\mathrm{ICC}_{\mathrm{V}}$ value for the 3D motion capture system was higher for all degrees of freedom, and the ROM measurements with the motion capture system met the reliability criterion for all joints except for the PIP3.

Overall, $43 \%$ and $67 \%$ of the goniometric measurements had a test-retest difference below $5^{\circ}\left(\mathrm{PD}_{\mathrm{G}}<5^{\circ}\right)$ and $10^{\circ}\left(\mathrm{PD}_{\mathrm{G}}<10^{\circ}\right)$, respectively (Table 2$)$. The corresponding percentages for the $3 \mathrm{D}$ motion capture system were $67 \% \quad\left(\mathrm{PD}_{\mathrm{V}}<5^{\circ}\right)$ and $92 \% \quad\left(\mathrm{PD}_{\mathrm{V}}<10^{\circ}\right)$, respectively. The DIP5 was the only joint for which slightly more volunteers had an angular difference of less than $5^{\circ}$ for the goniometric measurements compared to the 3D motion capture system. For all other joints, the 3D motion capture system had a higher percentage of individuals with small $\left(<5^{\circ}\right)$ inter-session differences.

\section{Discussion}

In this study, we assessed the repeatability of ROM measurements of the hand joints with $3 \mathrm{D}$ motion capture system, compared them with manual goniometry, and calculated the MDD for both methods. We measured all joints of the fingers and the wrist.

We observed method differences from -57 to $+11^{\circ}$, where negative values indicate higher ROM when examined with the goniometer $\left(\mathrm{Mean}_{\text {romG }}>\mathrm{Mean}_{\text {romv }}\right)$.

Since the true value of the joint angle is unknown, the comparison between the two methods serves as the first step in the validation of the new motion analysis protocol. Skin movement relative to the bone is the biggest source of error in motion analysis with skin markers $[29,30]$. Longitudinal rotations are more affected from skin movement artifacts, therefore leading to an under-/overestimation of the joint angle in motion analysis [30, 31]. In agreement, we found the highest method disagreement $\left(57^{\circ}\right)$ and the only significant difference for the pronation-supination movement. Difficulties to measure the radioulnar joint with a goniometer (ICC $0.35_{\mathrm{G}}, \mathrm{SEM}_{\mathrm{G}} 6.8^{\circ}$ ) might have further contributed to the large difference between the methods.

Armstrong et al. suggest that the lack of precision in goniometric measurement could be technique related, as the current method of measuring true forearm rotation involves placing a flat goniometer along the curved surface of the flexion/extension crease of the wrist [32]. The observed significant difference between the methods for measuring forearm rotation indicates that adjustments to the methodology are necessary. Schmidt et al. propose a procedure to reduce the influence of skin movement artifacts by looking at the hand rotation during pronation-supination instead of the rotation of the forearm itself [33]. Implementation of such simple corrections might further improve the presented measurement method. 
A comparison with a gold standard, such as an imaging technique, would be a possibility to estimate the accuracy of the measurements. However, the validity of the data is beyond the scope of this article. Still, our results reveal that the measurement system used to obtain the ROM has to be considered for clinical data interpretation. Therefore, a specific norm database for every method is highly recommended.

$\mathrm{n}$ a clinical setting, methods are often used to evaluate the effects of interventions or monitor changes over time within the same subject. Therefore, a focus on agreement parameters is recommended by de Vet et al. [26]. The SEM and MDD express measurement error in the same unit as the original value, which facilitates clinical interpretation. In contrast to ICC, SEM and MDD are not influenced by variability among the sample [34]. Hence, their values can be transferred to various groups of patients.

Averaged over all analyzed joints, the $\mathrm{MDD}_{\mathrm{V}}$ was $10^{\circ}$, compared to $18^{\circ} \mathrm{MDD}_{\mathrm{G}}$. Therefore, measurements by means of a motion capture system allow us to recognize smaller changes in joint mobility than with goniometer. This means that we have to be very careful in the clinical setting to interpret a change in the ROM as a true change, or just as a measurement error. For the wrist joint flexion/extension and radial/ulnar deviation, $\mathrm{MDD}_{\mathrm{G}}$ was $18^{\circ}$. Macedo and Magee examined the passive ROM of the wrist with a universal goniometer in 12 healthy adults. They found a $M D D_{G}$ for the wrist flexion of $11^{\circ}$ and for the wrist extension of $8^{\circ}$, which is lower than our $\mathrm{MDD}_{\mathrm{G}}$, but higher than our $\mathrm{MDD}_{\mathrm{V}}$ [35].

The $\mathrm{MDD}_{\mathrm{G}}$ for the finger joint lied between $12^{\circ}$ and $24^{\circ}$. Ellis and Bruton examined the finger joints with a goniometer, but in a splinted position, so they had MDD (reported as 95\% confidence interval of difference) of between $4^{\circ}$ and $5^{\circ}$ [1].

Overall, the calculated precision of the ROM measurements was $\mathrm{SEM}_{\mathrm{V}} 3.6^{\circ}$ and $\mathrm{SEM}_{\mathrm{G}} 6.4^{\circ}$ for the 3D motion capture system and goniometer, respectively. The mean values of all repeatability parameters indicate higher test-retest agreement for the 3D motion capture method. For the wrist joint, the $\mathrm{SEM}_{\mathrm{G}}$ with the goniometer was $6.4^{\circ}(\mathrm{F} / \mathrm{E})$ and $6.5^{\circ}(\mathrm{R} / \mathrm{U})$. LaStayo and Wheeler assessed the passive ROM of the wrist with a universal goniometer in 120 patients with wrist conditions. They reported SEM between $5.6^{\circ}$ and $8.1^{\circ}$, like Macedo and Magee with SEM between $2.9^{\circ}$ and $7.4^{\circ}$, compare to Horger who calculated a SEM between $2.6^{\circ}$ and $4.5^{\circ}[12,35,36]$. Our results consider the ROM of the movement, so both measurement points (e.g., maximum flexion and extension position) are affected from independent error associated with the placement of the goniometer, whereas the other studies showed the results of each direction separately.

For the finger joints, $\mathrm{SEM}_{\mathrm{G}}$ was between $4.2^{\circ}$ and $8.8^{\circ}$. Stam et al. evaluated 20 healthy subjects with a goniometer while holding cylinders with different diameter and had a SEM between $4^{\circ}$ and $6^{\circ}$, similar to our results [37].

In comparison with previous repeatability goniometry studies, the intrarater reliability for the active ROM of the middle finger found in our study lies within the range of the intra- and interrater reliability (ICC 0.43 to 0.99 ) determined by Lewis et al. [2] Solgaard et al. assessed intraobserver SDD for the goniometry of the wrist of $5.2-8^{\circ}$ [38]. Compared to these findings, our results for wrist goniometry are slightly higher. In contrast, the $3 \mathrm{D}$ motion capture of the wrist ROM had better repeatability than the goniometry results in both studies.

Compared to previous measurements by $3 \mathrm{D}$ motion capture, we found excellent test-retest reliability on the wrist (ICC 0.90-0.97). The corresponding values in Levanon et al. were only good (ICC 0.770.83) [39]. In contrast, the root-mean-square error in our study was $5.3^{\circ}$, whereas Sancho-Bru et al. found smaller errors in repeatability $\left(3.4^{\circ}\right)$ [8]. In that study, a different marker set was used and the repeatability was assessed for grasping different objects while we analyzed the maximum ROM. It is possible that variability of the ROM movement is bigger than in specific grasping tasks, but to quantify the source of error, a validation would be needed.

\section{Limitations and achievements}

In this study, we do not simply compare two different measurement tools but rather two different measurement procedures. Therefore, the comparison includes methodological differences in addition to the measurement system itself, which might have influenced the reported repeatability.

In manual goniometry, every joint was assessed separately. The kinematic analysis resulted in a continuous angular curve, from which the ROM was extracted. In comparison, the goniometer had to be placed twice for each joint in order to obtain the ROM, always in an interaction with the subject, which can influence the result. Hence, both measurement points (e.g., maximum flexion and extension position) are affected from independent error associated with the placement of the goniometer, whereas the position of the skin markers stayed the same during the whole session (compensation of error possible). We do not see this as a limitation of the 
study, but rather as an advantage of the motion analysis method.

The main difference between the two protocols was the amount of measurements of each joint angle. When using the motion capture system, the maximum ROM of the dynamic trials could be averaged within the session, which might have compensated for outliers. In contrast, with the manual goniometer, each parameter was measured only once per session, as otherwise the rater could recall the values. This difference has likely contributed to the better results for the 3D motion capture system. Still, the study implements both methods such as they are usually applied in a clinical setting. It quantifies the test-retest repeatability of a realistic application, where usually a single surgeon or therapist measures the ROM to monitor change during treatment. We are aware that we cannot make a statement about the accuracy of both methods, the 3D motion capture system and the goniometry. A comparison with a radiological examination would be necessary for this. As a first step, we concentrated on the repeatability of both methods and on their comparison, since repeated tests to assess changes are very common in the clinical setting and the research [26].

We missed few measurements because of marker loss. This could happen while they are attached only with a double-sided adhesive tape, and can get lost or displaced, which is a disadvantage in motion analysis. The advantage of the 3D motion capture system is the dynamic evaluation of the wrist and all finger joints simultaneously. Therefore, it can be applied for the assessment of the ROM as well as dynamic functional tasks, such as activities of daily living. The main advantages of the manual goniometry are that it is much easier to implement in the clinical setting. Our study shows that in applications where the goniometer is not precise enough, motion analysis is a possible alternative due to its lower MDD. The choice of the method has to be in accordance with the research question and the expected or clinically relevant change in joint ROM.

\section{Conclusion}

In conclusion, the MDD of the 3D motion capture system is smaller than of the goniometer measurement. This is particularly important in an experimental setup where a higher degree of precision is requested. In the clinical research, better MDD permits relevant reduction of the sample size.

\section{Abbreviations}

DIFF: Test-retest difference; DIP: Distal interphalangeal joint; ICC: Intraclass correlation coefficient; IP: Interphalangeal joint; MCP: Metacarpophalangeal joint; MDD: Minimal detectable difference; PIP: Proximal interphalangeal joint; ROM: Range of motion; SEM: Standard error of measurement

\section{Acknowledgements \\ We would like to thank Vera Beckmann and the Hand Therapy Department of the University Hospital of Zurich for their support in this study.}

\section{Funding}

This research received no specific grant from any funding agency in public, commercial, or not-for-profit sectors.

\section{Availability of data and materials}

The datasets generated and analyzed during the current study are available in the password-protected System of the University Hospital Zurich repository. The datasets used and/or analyzed during the current study are available from the corresponding author on reasonable request.

\section{Authors' contributions}

$L R$, GF, and RL captured, analyzed, and interpreted the data and wrote the manuscript. MC and PG supervised and created parts of the manuscript. WRT wrote part of the manuscript and corrected it. MC was the principal investigator of the study and acted as a supervisor of LR. All authors read and approved the final manuscript.

\section{Ethics approval and consent to participate}

The study is approved by the Ethical Committee Zurich (Cantonal Ethical Committee number: Kek-ZH-Nr: 2015-0395).

Contact: Ethical Committee Zurich, Stampfenbachstrasse 121, 8090 Zurich

E-mail: Info.KEK@kazzh.ch

Tel: +41 432597970

Lisa Reissner, Gabriella Fischer, Renate List, William R. Taylor, Pietro Giovanoli, and Maurizio Calcagni approved the human protocol for this investigation that all investigations were conducted in conformity with ethical principles of research according to the declaration of Helsinki and that informed consent for participation in the study was obtained.

\section{Consent for publication}

Not applicable.

\section{Competing interests}

The authors declare that they have no competing interests.

\section{Publisher's Note}

Springer Nature remains neutral with regard to jurisdictional claims in published maps and institutional affiliations.

\section{Author details}

'Division of Plastic Surgery and Hand Surgery, University Hospital Zurich, University of Zurich, Raemistrasse 100, 8091 Zurich, Switzerland. ${ }^{2}$ Institute for Biomechanics, ETH Zurich, Zurich, Switzerland. ${ }^{3}$ Human Performance Lab, Schulthess Clinic, Zurich, Switzerland.

Received: 5 January 2019 Accepted: 30 April 2019

Published online: 10 June 2019

\section{References}

1. Ellis B, Bruton A. A study to compare the reliability of composite finger flexion with goniometry for measurement of range of motion in the hand. Clin Rehabil. 2002;16:562-70.

2. Lewis $E$, Fors $L$, Tharion WJ. Interrater and intrarater reliability of finger goniometric measurements. Am J Occup Ther. 2010;64:555-61.

3. Chiu HY, Su FC, Wang ST, Hsu HY. The motion analysis system and goniometry of the finger joints. J Hand Surg Br. 1998;23:788-91.

4. Kuo LC, Su FC, Chiu HY, Yu CY. Feasibility of using a video-based motion analysis system for measuring thumb kinematics. J of Biomech. 2002;35: 1499-506.

5. Rash GS, Belliappa PP, Wachowiak MP, Somia NN, Gupta A. A demonstration of validity of 3-D video motion analysis method for measuring finger flexion and extension. J of Biomech. 1999:32:1337-41.

6. Coupier J, Hamoudi S, Telese-Izzi S, Feipel V, Rooze M, Van Sint Jan S. A novel method for in-vivo evaluation of finger kinematics including 
definition of healthy motion patterns. Clin Biomech (Bristol, Avon). 2016;31: 47-58.

7. Degeorges R, Parasie J, Mitton D, Imbert N, Goubier JN, Lavaste F. Threedimensional rotations of human three-joint fingers: an optoelectronic measurement. Preliminary results. Surg Radiol Anat. 2005;27:43-50.

8. Sancho-Bru JL, Jarque-Bou NJ, Vergara M, Pérez-Gonzalez A. Validity of a simple videogrammetric method to measure the movement of all hand segments for clinical purposes. Proc Inst Meech Eng H. 2014;228:182-9.

9. Ellis B, Bruton A, Goddard JR. Joint angle measurement: a comparative study of the reliability of goniometry and wire tracing for the hand. Clin Rehabil. 1997;11:314-20.

10. Groth HG, Ehretsman RL. Goniometry of the proximal and distal interphalangeal joints, Part l: a survey of instrumentation and placement preferences. J Hand Ther. 2001;14:18-22.

11. Hamilton GF, Lachenbruch PA. Reliability of goniometers in assessing finger joint angle. Phys Ther. 1969;49:465-9.

12. LaStayo PC, Wheeler DL. Reliability of passive wrist flexion and extension goniometric measurements: a multicenter study. Phys Ther. 1994;74:162-76.

13. Boone DC, Azen SP, Lin CM, Spence C, Baron C, Lee L. Reliability of goniometric measurements. Phys Ther. 1978:58:1355-60.

14. Mayerson NH, Milano RA. Goniometric measurement reliability in physical medicine. Arch Phys Med Rehabil. 1984;65:92-4.

15. Wise $\mathrm{S}$, Gardner W, Sableman E, et al. Evaluation of a fiber optic glove for semi-automated goniometric measurements. J Rehabil Res Dev. 1990;27: 411-24.

16. Cook JR, Baker NA, Cham R, Hale E, Edfern MS. Measurements of wrist and finger postures: a comparison of goniometric and motion capture techniques. J Appl Biomech. 2007;23:70-8.

17. Norkin C, White J. Measurement of joint motion: a guide to goniometry. Fifth ed. Philadelpihia: F.A. Davis; 2016.

18. Casanova J. ASHT clinical assessment recommendations. 3rd ed. Chicago: American Society of Hand Therapists; 2013.

19. Engstrand C, Krevers B, Kvist J. Interrater reliability in finger joint goniometer measurement in Dupuytren's disease. Am J Occup Ther. 2012;66:98-103.

20. Metcalf CD, Notley SV, Chappell PH, Burridge JH, Yule VT. Validation and application of a computational model for wrist and hand movements using surface markers. IEEE Trans Biomed Eng. 2008;55:1199-210.

21. Metcalf CD, Notley SV. Modified kinematic technique for measuring pathological hyperextension and hypermobility of the interphalangeal joints. IEEE Trans Biomed Eng. 2011;58:1224-31.

22. List R, Gülay T, Stoop M, Lorenzetti S. Kinematics of the trunk and the lower extremities during restricted and unrestricted squats. J strength Cond Res. 2013;27:1529-38

23. Grood ES, Suntay WJ. A joint coordinate system for the clinical description of three-dimensional motions: application to the knee. J Biomech Eng. 1983:105:136-44

24. Wu G, van der Helm FC, Veeger HE, Makhsous M, Van Roy P, Anglin C, Nagels J, Karduna AR, McQuade K, Wang X, Werner FW, Buchholz B. International Society of Biomechanics. ISB recommendation on definitions of joint coordinate systems of various joints for the reporting of human joint motion - Part II: shoulder, elbow, wrist and hand. J Biomech. 2005;38: 981-92.

25. Streiner DL, Gr N. Health measurement scales: a practical guide to their development and use. New York: Oxford University Press; 2003.

26. De Vet $H C$, Terwee $C B$, Knol DL, Bouter LM. When to use agreement versus reliability measures. J Clin Epidemiol. 2006;59:1033-9.

27. Weir JP. Quantifying test-retest reliability using the intraclass correlation coefficient and the SEM. J Strength Cond Res. 2005;19:231-40.

28. Clarkson H. Musculoskeletal assessment (2nd ed). Philadelphia, PA: Lippincott, Williams\&Wilkins.

29. Cappozzo A, Catani F, Leardini A, et al. Position and orientation in space of bones during movement: experimental artefacts. Clin Biomech. 1996:11:90-100.

30. Leardini A, Chiari L, Della Croce U, et al. Human movement analysis using stereophotogrammetry Part 3. Soft tissue artifact assessment and compensation. Gait and Posture. 2005;21:212-25.

31. Cappozzo AF, Catani F, Croce UD, Leardini A. Position and orientation in space of bones during movement: anatomical frame definition and determination. Clin Biomech. 1995;10:171-8.
32. Amstrong AD, MacDermid JC, Chinchalkar S, Stevens RS, King GJ. Reliability of range-of-motion measurement in the elbow and forearm. J Shoulder Elbow Surg. 1998;7:573-80.

33. Schmitd R, Disselhorst-Klug C, Silny J, Rau G. A marker-based measurement procedure for unconstrained wrist and elbow motions. J Biomech. 1999;32: 615-21.

34. Stratford PW, Goldsmith CH. Use of the standard error as a reliability index of interest: an applied example using elbow flexor strength data. Phys Ther. 1997;77:745-50.

35. Macedo LG, Magee DJ. Effects of age on passive range of motion of selected peripheral joints in healthy adult females. Physiother Theory Pract. 2009;25:145

36. Horger MM. The reliability of goniometric measurements of active and passive wrist motions. Am J Occup Ther. 1990;44:342.

37. Stam HJ, Ardon MS, Den ouden AH, Schreuders TAR, Roebroeck ME. The compangle: a new goniometer for joint angle measurements of the hand. Eur Medicophys. 2006:42:37.

38. Solgaard S, Carlsen A, Kramhoft M, Petersen VS. Reproducibility of goniometry of the wrist. Scand J Rehabil Med. 1986;18:5-7.

39. Levanon Y, Gefen A, Lerman Y, Givon U, Rathon NZ. Validity and reliability of upper extremity three-dimensional kinematics during a typing task. Gait Posture. 2010:32:469-74.

\section{Ready to submit your research? Choose BMC and benefit from:}

- fast, convenient online submission

- thorough peer review by experienced researchers in your field

- rapid publication on acceptance

- support for research data, including large and complex data types

- gold Open Access which fosters wider collaboration and increased citations

- maximum visibility for your research: over $100 \mathrm{M}$ website views per year

At BMC, research is always in progress.

Learn more biomedcentral.com/submissions 